\title{
Orthostatic hypotension associated with paroxysmal ventricular tachycardia
}

\author{
R. N. NANDA AND R. H. JOHNSON \\ From the University Department of Neurology, Institute of Neurological Sciences, \\ Southern General Hospital, Glasgow
}

SYNOPSIS Two patients (aged 46 and 49 years) are presented who gave a history of several years' duration of unsteadiness, dizziness, and syncopal attacks on standing. Both had orthostatic hypotension which was associated with the development of a unifocal paroxysmal ventricular tachycardia. There was no evidence of organic heart disease. In one of the patients the symptoms usually developed when standing after working in a crouched position. He responded to treatment with $\beta$ adrenergic blockade. The other patient developed her symptoms on standing, after exercise or other stress. The paroxysmal ventricular tachycardia, which occurred in the upright position only, was accompanied by a marked rise in plasma adrenaline. In this patient one contributory factor was a low blood volume and she responded to plasma volume expansion. We wish to draw attention to the common neurological symptoms with which paroxysmal ventricular tachycardia may present. We suggest that paroxysmal ventricular tachycardia may result from ventricular sensitivity to circulating adrenaline and not due to aberrant innervation of the heart as has been suggested previously.

Orthostatic hypotension is being recognized increasingly as a complication of many neurological disorders. It may occur idiopathically or be secondary to well-recognized diseases. In both situations baroreceptor reflex pathways are affected. The disorders have recently been reviewed (Johnson and Spalding, 1974). We describe two patients who presented to a neurological clinic with dizziness and syncope on standing. Although orthostatic hypotension was observed, neither patient showed any evidence of involvement of autonomic reflexes or other evidence of neurological disease. In both patients the orthostatic hypotension was found to be related to paroxysmal ventricular tachycardia in the absence of underlying organic heart disease. Paroxysmal ventricular tachycardia usually occurs in patients with severe heart disease (Armbrust and Levine, 1950), but in a small proportion of patients it occurs in subjects with no evidence of heart disease (Hermann et al., 1959; Lesch et al., 1967). Both patients have

(Accepted 17 February 1975.) 698 been successfully treated, one with a $\beta$-adrenergic receptor blocker, practolol, and the other with 9- $\alpha$-fluorohydrocortisone. The pathogenesis and treatment of this form of cardiac dysrhythmia are discussed.

\section{CASE 1}

P.O'B. (Institute of Neurological Sciences, Glasgow, No. 803735) was a 46 year old man employed as a tunnel miner. He was referred by his general practitioner because of a two years' history of dizziness and unsteady gait, progressing on occasion to loss of consciousness. He was well in the early part of each day but, after working in a crouched position, often felt light-headed and unsteady on standing, and throughout the evening he was frequently unsteady for a few seconds at a time. The attacks also occurred at work if he came to a shaft in a tunnel and stood up. He had always had good health in the past and had no abnormality of bladder, bowel, or sexual function. He drank beer in moderation and did not smoke. On examination his height was $172 \mathrm{~cm}$ and weight $70 \mathrm{~kg}$. There was marked orthostatic hypotension, the lying blood pressure of $120 / 70 \mathrm{mmHg}$ falling on standing after two minutes to $70 \mathrm{mmHg}$ 
systolic pressure, the diastolic pressure being at an unrecordable level. He began overbreathing when the blood pressure fell, sweated, and became pale and there was a tachycardia (about 120 beats/min). However, the orthostatic hypotension was variable and at several examinations was not present. The rest of the examination was unremarkable.

\section{CASE 2}

E.D. (I.N.S., No. 812439) was a 49 year old female medical practitioner. Since childhood the patient had experienced attacks of weakness and unsteadiness particularly when standing. Sometimes there was associated nausea and vomiting. The symptoms were episodic and appeared to be aggravated by stress of any kind and by exercise or warm weather. She had frequently had periods of several weeks or months in which she was more prone to develop attacks. At such times the attacks could be provoked by sitting up in bed or standing and loss of consciousness could occur. At times the symptoms were particularly bad after getting out of bed in the morning. The symptoms were so protracted that she had been seen by many physicians including neurologists with negative results. Bladder and bowel function were normal, alcohol consumption was nil, and she did not smoke. On examination her height was $167 \mathrm{~cm}$ and her weight was $58 \mathrm{~kg}$. Her blood pressure when supine was $130 / 80 \mathrm{mmHg}$ and when she stood there was no immediate fall of blood pressure, but after 15 minutes or more the systolic blood pressure was $60 \mathrm{mmHg}$ and the diastolic pressure was unrecordable. The heart rate was initially 65 beats $/ \mathrm{min}$ but, as she remained standing, the heart rate became irregular, and increased to over 160 beats $/ \mathrm{min}$ with coupling. When the blood pressure fell she became cold and clammy and fainted on one occasion. No other abnormalities were found in the remainder of the examination.

\section{INVESTIGATIONS}

The following investigations were done on both patients with normal results: blood count and film, ESR, WR (blood), serum $B_{12}$ and folate, blood sugar and urea, plasma protein bound iodine, serum electrolytes, urinary metadrenaline $(24 \mathrm{~h})$ and porphyrins, chest radiograph, electroencephalogram, and brain scan.

TESTS OF AUTONOMIC NERVOUS SYSTEM FUNCTION In both patients the blood pressure was recorded with an intra-arterial needle and a capacitance manometer system. A normal response of both blood pressure and heart rate was obtained to mental arithmetic, noise, and an acute rise in intrathoracic pressure (Valsalva's manoeuvre) indicating that nervous reflexes subserving blood pressure regulation were normal. The patients were warmed with a heat cradle and sweating occurred normally over face, trunk, and extremities. Iontophoresis of acetylcholine produced normal sweating and piloerection (Macmillan and Spalding, 1969). The latter tests indicate normal activity of other sympathetic nerve fibres.

TESTS OF CARDIAC FUNCTION AND BLOOD VOLUME In case 1 , the initial electrocardiogram (ECG) in the horizontal position showed sinus rhythm with a heart rate of $68 / \mathrm{min}$ and there were occasional unifocal ventricular ectopic beats. He was tested on a table tilted to $85^{\circ}$ head-up while a continuous ECG was obtained. The ventricular ectopic beats became more frequent in the erect posture (Fig. 1). The

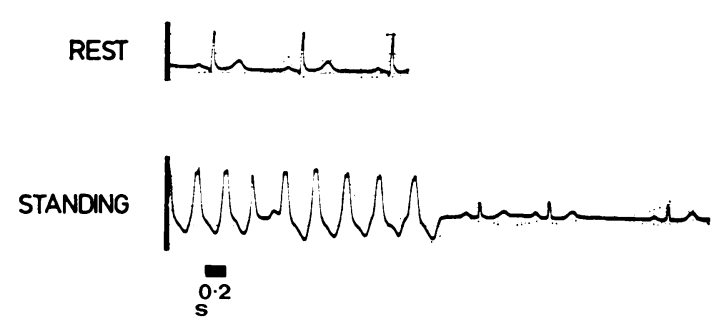

FIG. 1 Case 1. Electrocardiograms at rest (supine) and on standing for two minutes, showing a paroxysm of ventricular tachycardia in the erect position.

blood pressure of $120 / 70 \mathrm{mmHg}$ when he was horizontal fell after two minutes tilting to $60 \mathrm{mmHg}$ systolic pressure and he fainted. He was immediately returned to the horizontal position and regained consciousness after about two minutes. When the procedure was repeated after treatment with the $\beta$-adrenergic receptor blocker practolol there was no change of blood pressure or cardiac rhythm on standing erect. Plasma volume estimated with a radioactive iodine labelled human serum albumin method (RIHSA), when he was asymptomatic was normal (3 $470 \mathrm{ml})$.

In case 2, an ECG before orthostatic hypotension had been observed showed occasional ectopic beats which were interpreted as benign with no evidence of cardiac disease. She was examined on a tilt table in the horizontal position and was then tilted to $85^{\circ}$, 


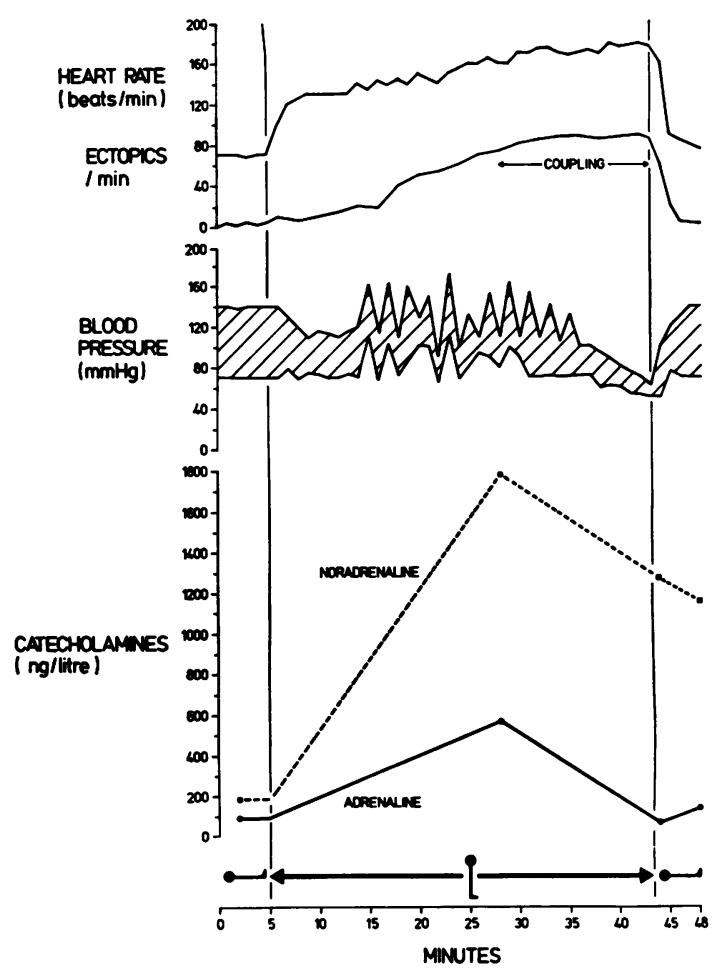

FIG. 2 Case 2. The effect of standing for 38 minutes on heart rate, ventricular ectopic beats, blood pressure, and plasma catecholamines. The period of tilt at $85^{\circ}$ is shown above the horizontal axis.

observation being continued (Fig. 2). An intraarterial blood pressure recording was obtained in the horizontal and erect positions. Venous blood was obtained for estimation of blood and plasma volume (RIHSA) and circulating adrenaline and noradrenaline (Valori et al., 1970, modified by Rennie, 1974). The supine blood pressure was $140 / 70 \mathrm{mmHg}$ and the heart rate $68-70$ beats $/ \mathrm{min}$ with no ectopic beats. After two minutes in the tilted position the blood pressure fell to $115 / 70 \mathrm{mmHg}$, the heart rate increased to 120 beats/min, and there were occasional ventricular ectopic beats. The heart rate continued to rise and the ectopic beats became more frequent; blood pressure fiuctuated from $130 / 85$ to $110 / 75$ $\mathrm{mmHg}$ from beat to beat and the changes during this period are shown diagrammatically in Fig. 2. After 27 minutes, the heart rate was 170/min with unifocal ventricular ectopic beats occurring after each normal heart beat. The blood pressure fell to $62 / 45 \mathrm{mmHg}$ by 38 minutes; the patient felt faint and nauseated and was returned to the horizontal position. Within one to two minutes the ectopic beats became less frequent and the blood pressure started rising. Ten minutes later blood pressure was $150 / 90 \mathrm{mmHg}$ and the heart rate $84 / \mathrm{min}$ with only the occasional ectopic beat. Values of circulating plasma adrenaline and noradrenaline were normal when the patient was in the horizontal position, and rose considerably in the sample taken towards the end of the period of tilting. On return to the horizontal position, the adrenaline values fell rapidly to normal, while the noradrenaline concentration remained high (Fig. 2; Table). Estimation of blood volume in the horizontal position showed a deficit from the predicted normal (Table). The tipping

TABLE

CASE 2: PlASMA CATECHOLAMINES AND BLOOD VOLUMES

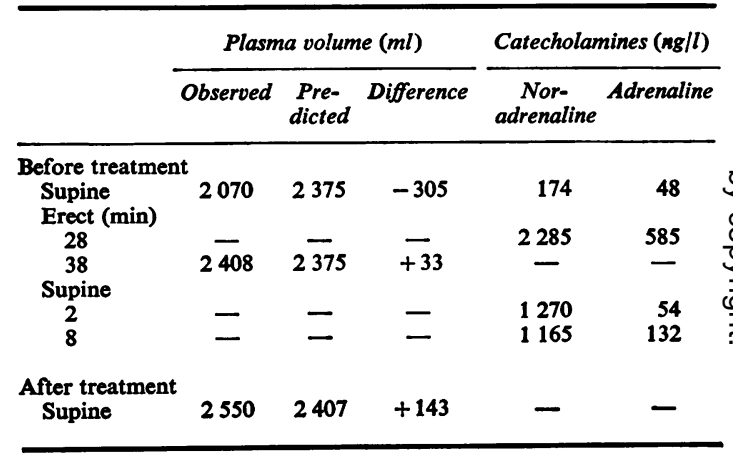

procedure was repeated on another occasion before and after an albumin infusion (30 $\mathrm{g}$ albumin in $\mathbf{8 0 0}$ $\mathrm{ml} \mathrm{5 \%}$ dextrose in 16 minutes) to increase the plasma volume. After the infusion of albumin there was no change of blood pressure or heart rate on standing erect for $\mathbf{4 5}$ minutes and the patient remained symptomless. Investigations of the low plasma volume failed to reveal a cause. There was, however, normal sodium conservation during a period of low sodium intake and a normal reninangiotensin-aldosterone system. Baseline values of plasma angiotensin and aldosterone were $15 \mathrm{pg} / \mathrm{ml}$ and $9.8 \mathrm{ng} / \mathrm{dl}$ respectively rising during a five day period in which sodium intake was reduced (10 $\mathrm{mmol} /$ day) to $30 \mathrm{pg} / \mathrm{ml}$ and $23 \mathrm{ng} / \mathrm{dl}$ respectively.

TREATMENT Case 1 was treated with practolol (100 mg thrice daily) with benefit. His symptoms became less marked but were not abolished com- 
pletely. He was able to lead a relatively normal life but did not return to his old job. In view of the successful response to acute expansion of plasma volume case 2 was treated with 9- $\alpha$-fluorohydrocortisone. After the third day of treatment she was able to stand for as long as she wished. No fall of blood pressure or rise in pulse rate or change in ECG was observed when the tilt table procedure was repeated 11 days after starting the therapy, and a week later she was able to walk and climb stairs normally. She has been able to return to her occupation.

\section{DISCUSSION}

Both patients presented to a neurological department with symptoms of orthostatic hypotension but no evidence of an anatomical lesion of autonomic pathways was found. The possibility that the tachycardia was initiated by orthostatic hypotension due to autonomic failure is therefore ruled out and another cause must be considered. It has been suggested that these patients may have an aberrant parasympathetic innervation of the heart (Jick and Smith, 1963). We now report evidence, however, that the condition when fully developed is related to abnormally high levels of circulating catecholamines and the condition is therefore more likely to be related to these than to aberrant innervation.

In case 1 the fall of blood pressure in the standing position was associated with the development of runs of ventricular tachycardia. The arrhythmia was more liable to occur when he stood erect after exercise. In case 2 a contributing stress factor was the low plasma volume. The initial drop of systolic blood pressure of $10-20 \mathrm{mmHg}$ on standing was possibly due to the low plasma volume. In case 2 more marked hypotension occurred after 15-30 minutes of standing as a severe ventricular tachycardia developed due to unifocal ventricular ectopic beats. Thus in both patients there was gross ventricular tachycardia which has been shown experimentally to result in fall of cardiac output and of blood pressure (Wégria et al., 1958). In case 2 there was at the same time a marked increase in the levels of circulating adrenaline and noradrenaline. It seems possible that the initial fall of blood pressure caused a rise in plasma catecholamines which acted on a sensitive ventricular focus resulting in an ectopic rhythm.
The adrenaline values returned to normal with the patient in the horizontal position and ventricular ectopic beats ceased. Noradrenaline concentrations fell more slowly. The sensitivity of the ventricular ectopic beats would therefore appear to be due to the adrenaline rather than noradrenaline. The cause of the low plasma volume in case 2 is difficult to explain. Investigations showed that it was not due to a failure of sodium conservation because of abnormalities within the renin-angiotensin-aldosterone system. One possible factor contributing to the low plasma volume may be that the patient had been very inactive, spending much of her time in bed for the preceding nine months (Dietrick et al., 1948), but nevertheless she had previously had symptoms during active periods of her life. In both patients there was no evidence of organic heart disease to account for the paroxysmal ventricular dysrhythmia.

Paroxysmal ventricular tachycardia, provoked by exertion or excitement, in the absence of organic heart disease is a rare condition well described by Wilson et al. (1932). Peters and Penner (1946) described a patient in whom the attacks of paroxysmal ventricular tachycardia appeared in the upright position only, and in this position were precipitated by excitement or exertion. These precipitating causes are further support for a conclusion that the condition can be due to unusual sensitivity of the heart to adrenergic stimulation. Jick and Smith (1963) reported a case in which exercise did not abolish the pulse bigeminy, whereas atropine did, and they postulated that the patient had anomalous vagal innervation of the ventricular myocardium. More recently, Lesch et al. (1967) reported one case and reviewed the English literature. In their patient, symptoms occurred when resting, after a strenuous day, and on several occasions syncopal attacks had occurred. The ECG showed frequent premature ventricular contractions which could be abolished by mild exercise. They concluded that the activity of the ectopic focus was rate dependent. In their review of 34 patients in the literature, they observed that each patient presented a completely erratic course, and, although the disorder is usually benign, the occurrence of sudden death in some of these patients dictates that an effective therapeutic programme needs to be established in each case. 
Beta-adrenergic blockade has been used successfully in one study (Taylor and Halliday, 1965), as in our experience with case 1 . This would support the suggestion that sympathetic stimuli are important in producing the arrhythmia. In case 2 , this is suggested in our study by the correlation of the development of cardiac irregularity with high levels of circulating catecholamines, particularly adrenaline. Therapy with $\beta$-adrenergic receptor blockade has not yet been tried in this patient because of the favourable response to volume expansion with 9- $\alpha-$ fluorohydrocortisone.

We wish to draw attention to this disorder as a cause of orthostatic hypotension which may lead to patients presenting at a neurological clinic. Our results suggest that paroxysmal ventricular tachycardia may develop because of sensitivity of the myocardium to high levels of circulating adrenaline and not because of aberrant innervation as has been suggested in some other cases.

We wish to thank the patients for their cooperation and the M.R.C. Blood Pressure Unit, Western Infirmary, Glasgow, for studies of the renin-angiotensin-aldosterone system. Professor J. A. Simpson gave encouragement. We are grateful to the National Fund for Crippling Diseases, the Migraine Trust, Roche Products Ltd, and the Scottish Home and Health Department for financial support.

\section{REFERENCES}

Armbrust, C. A., Jr, and Levine, S. A. (1950). Paroxysmal ventricular tachycardia: a study of one hundred and seven cases. Circulation, 1, 28-40.

Dietrick, J. E., Whedon, G. D., and Shorr, E. (1948). Effects of immobilization upon various metabolic and physiologic functions of normal men. American Journal of Medicine, 4, 3-36.

Hermann, G. R., Park, H. M., and Hejtmancik, M. R. (1959). Paroxysmal ventricular tachycardia: a clinical and electrocardiographic study. American Heart Journal, 57, 166-176.

Jick, H., and Smith, J. E. (1963). An unusual case of bigeminy due to vagal tone. American Journal of Cardiology, 12, 741-744.

Johnson, R. H., and Spalding, J. M. K. (1974). Disorders of the Autonomic Nervous System. Blackwell: Oxford.

Lesch, M., Lewis, E., Humphries, J. O'N., and Ross, R. S. (1967). Paroxysmal ventricular tachycardia in the absence of organic heart disease. Annals of Internal Medicine, 66, 950-960.

Macmillan, A. L., and Spalding, J. M. K. (1969). Human sweating response to electrophoresed acetylcholine: a test of postganglionic sympathetic function. Journal of Neurology, Neurosurgery, and Psychiatry, 32, 155-160.

Peters, M., and Penner, S. L. (1946). Orthostatic paroxysmal ventricular tachycardia. American Heart Journal, 32, 645652.

Rennie, M. J. (1974). Physiological and Biochemical Changes with Exercise. Thesis: University of Glasgow.

Taylor, R. R., and Halliday, E. J. (1965). Beta-adrenergic blockade in the treatment of exercise-induced paroxysmal ventricular tachycardia. Circulation, 32, 778-781.

Valori, C., Brunori, C. A., Renzini, V., and Corea, L. (1970). Improved procedure for formation of epinephrine and norephinephrine fluorophors by the trihydroxyindoles reaction. Analytical Biochemistry, 33, 158-167.

Wégria, R., Frank, C. W., Wang, H. H., and Lammerant, J (1958). The effect of atrial and ventricular tachycardia on: cardiac output, coronary blood flow and mean arterial blood pressure. Circulation Research, 6, 624-632.

Wilson, F. N., Wishart, S. W., Macleod, A. G., and Barker, P. S. (1932). A clinical type of paroxysmal tachycardia of ventricular origin in which paroxysms are induced by exertion. American Heart Journal, 8, 155-169. 